\title{
Empirical Mode Decomposition De-noising Method Based on Improved Soft-Threshold
}

\author{
Xi Jian-hui ${ }^{a}$, Chen Jiab
}

\author{
School of Automation, Shenyang Aerospace University, No.37 Daoyi South Avenue, Daoyi \\ Development District, Shenyang, China, 110136 \\ aE-mail: xjhui_01@163.com, 'E-mail: chenjia3018@163.com
}

\begin{abstract}
Keywords: Empirical Mode Decomposition (EMD); Intrinsic Mode Function (IMF); Threshold De-noising; Signal-to-Noise Ratio (SNR); Mean Square Error (MSE).
\end{abstract}

\begin{abstract}
In this paper, an improved soft-threshold function is constructed, combined the improved function and empirical mode decomposition (EMD) methods, a new de-noising method has been proposed. Set the adaptive threshold for the intrinsic mode functions (IMFs) of the EMD, and then de-noise the each IMF respectively. Finally, the de-noised signal is reconstructed by the de-noised IMF components. Through the simulation results of quantitative analysis by signal-to-noise ratio (SNR) and mean square error (MSE), the algorithm in this paper has better de-noising effect. Also, this method can effectively improve the constant deviation between the original signal and the de-noised signal by traditional soft-threshold.
\end{abstract}

\section{Introduction}

In the process of signal acquisition and transmission, signal will be noised inevitably. Therefore, de-noising the noisy signal and extract the original signal is an important step in signal processing.

Wavelet transform has the multi-resolution feature, and has the ability to characterize local signal characteristics in the time domain and frequency domain. With the development of wavelet analysis, the wavelet transform has been more widely used in the signal de-noising fields [1]. However, the signal which has relative greater noise amplitude may obtain poor de-noise effect by using the wavelet transform method.

In 1999, Chinese-American Norden E. Huang proposed a new signal analysis -empirical mode decomposition (EMD) method [2]. The method is essentially a steady signal processing. By using the method, real signal fluctuations or trends can be decomposed in different scales, produce a series of intrinsic mode functions (IMFs) with different characteristics. EMD method is based on the signal as the local characteristic time scale, the frequency of each IMF component concerned not only with the sampling frequency, but also varies with the signal itself, so that preserved the characteristics of the signal itself, experience has shown that the signal filtering is superior to the other signal processing methods. At the same time, the EMD method decomposed signal based on data's own time scale features, no need to pre-set any base function. Because the EMD method has more effective on nonlinear and non-stationary data decomposition, and can decompose signal in the space domain, so this method can be used to distinguish between noise and geophysical signals in the real effective signal. Compared with the wavelet method, this method has better time-frequency characteristics.

\section{EMD Method}

EMD method defined the decompose signal delay between adjacent peaks as the time scale and allow the signal to break through the screening to include a number of different time scales IMF, IMF must meet the following two conditions [3] :(a) The maximum point number and zero crossing number of the signal points equal to the minimum number of points or a difference of 1. (b) At any point, the average value defined by the envelope maximum points and minimum points is zero.

The steps of the EMD are as follows [3]:

(1) Find the local maximum value set $\mathrm{X}_{\max }$ and the minimum value set $\mathrm{X}_{\min }$ of the signal $\mathrm{X}(\mathrm{t})$; 
(2) Cubic spline interpolation according to the $X_{\max }$ and $X_{\min }$;

(3) According to the upper and lower envelope, find the local mean value $m_{11}(t)$ of the original data $\mathrm{X}(\mathrm{t})$, the difference value of the original signal and local min-max value denoted as $\mathrm{h}_{11}=\mathrm{X}(\mathrm{t})-\mathrm{m}_{11}(\mathrm{t})$;

(4) $X(t)$ replaced by $h_{11}$, repeat the three steps above until the screen twice the standard deviation (Eq. 1) of the results of the standard less than the set (usually between 0.2 to 0.3 ), we regard $h_{11}$ meet the requirements of the IMF component, and is an IMF component, we denote that $\mathrm{c}_{1}=\mathrm{h}_{\mathrm{lk}}$,

$$
\begin{aligned}
& \mathrm{r}_{1}=\mathrm{X}(\mathrm{t})-\mathrm{c}_{1}, \quad \mathrm{X}(\mathrm{t})=\mathrm{r}_{1} ; \\
& \mathrm{SD}=\sum_{\mathrm{t}=0}^{\mathrm{T}} \frac{\left|\left(\mathrm{h}_{1(\mathrm{k}-1)}(\mathrm{t})-\mathrm{h}_{1 \mathrm{k}}(\mathrm{t})\right)\right|^{2}}{\mathrm{~h}_{1(\mathrm{k}-1)}^{2}(\mathrm{t})} .
\end{aligned}
$$

(5) Repeat the four steps above until $r_{n}$ or $c_{n}$ smaller than a predetermined value, or the remaining $\mathrm{rn}(\mathrm{t})$ is a monotone function, EMD of the original signal is end . We obtain that

$$
X(t)=\sum_{i=1}^{n} c_{i}+\mathrm{r}_{n i}
$$

After the steps above, we obtain n-IMF components and a residual signal by the EMD of the original signal $\mathrm{X}(\mathrm{t})$, decomposition of the IMF model component represents the characteristics of different scales contained in the original signal.

\section{The New Wavelet Threshold Function}

Threshold Setting. During the threshold de-noising, the noise variance $\sigma$ and the threshold value $\lambda$ will be used inevitably, the calculation methods of which are briefly explained as follows.

The noise variance could be obtained by using function $\sigma \mathrm{j}=$ wnoisest $(\mathrm{C}, \mathrm{L}, \mathrm{S})$ in MATLAB, where $[\mathrm{C}, \mathrm{L}]$ is wavelet coefficients, $\mathrm{S}=1,2, \ldots, \mathrm{J}$, which is the decomposition level of the estimated wavelet coefficients, noise variances $\sigma$ can be obtained in the corresponding scales. [4],

There are a variety of methods about threshold selection; here we use Eq. 3 mentioned in reference

$$
\lambda_{j}=\sigma \sqrt{2 \ln N} / \ln (j+1)
$$

where $N$ is the number of sample, $j$ is the scale index; $\lambda_{j}$ can be adjusted accordingly based on the different detail coefficients on each scale, it is more flexible and can obtain better de-noising result than the traditional single threshold.

Threshold Function. The traditional hard-threshold and soft-threshold functions are shown in Eq. 4and Eq. 5 respectively [5].

$$
\begin{aligned}
& \hat{\omega}_{h}= \begin{cases}\omega & \text { if }|\omega| \geq \lambda ; \\
0 & \text { otherwise. }\end{cases} \\
& \hat{\omega}_{s}= \begin{cases}\omega-\lambda & \text { if } \omega \geq \lambda ; \\
0 & \text { if }|\omega|<\lambda ; \\
\omega+\lambda & \text { if } \omega \leq-\lambda .\end{cases}
\end{aligned}
$$


where $\omega$ is the wavelet decomposition coefficients; $\hat{\omega}_{s}$ and $\hat{\omega}_{h}$ are the estimated wavelet coefficients; $\lambda$ is the threshold value we set.

It should be noted that the wavelet coefficients, the absolute value of which is large, are decreased by the soft threshold function, the process result in some loss of high frequency information, which makes the reconstructed signal has a greater variance. This motivates us to introduce a new threshold function that is continuous around the threshold, and which can be adapted to the characteristics of the input signal. Based on these observations, we defined a new custom threshold function as Eq. 6.

$$
\hat{\omega}=\left\{\begin{array}{cc}
-\sqrt{(\omega-2 \lambda)^{2}-(3 \lambda)^{2}} & \hat{\omega}<-\lambda ; \\
0 & |\hat{\omega}| \leq \lambda ; \\
\sqrt{(\omega+2 \lambda)^{2}-(3 \lambda)^{2}} & \hat{\omega}>\lambda .
\end{array}\right.
$$

where $\omega$ is the wavelet decomposition coefficients; $\hat{\omega}$ is the estimated wavelet coefficients; $\lambda$ is the threshold value we set. Schematic diagram of the three threshold functions are shown in Fig. 1. From the improved threshold function above and Fig. 1, we can see clearly that $\hat{\omega}$ value of the new threshold function is between hard-threshold and soft-threshold function, we can obtain more detail information of the original signal by using Eq. 6, and also can avoid the oscillation phenomenon caused by discontinuity of the hard-threshold.

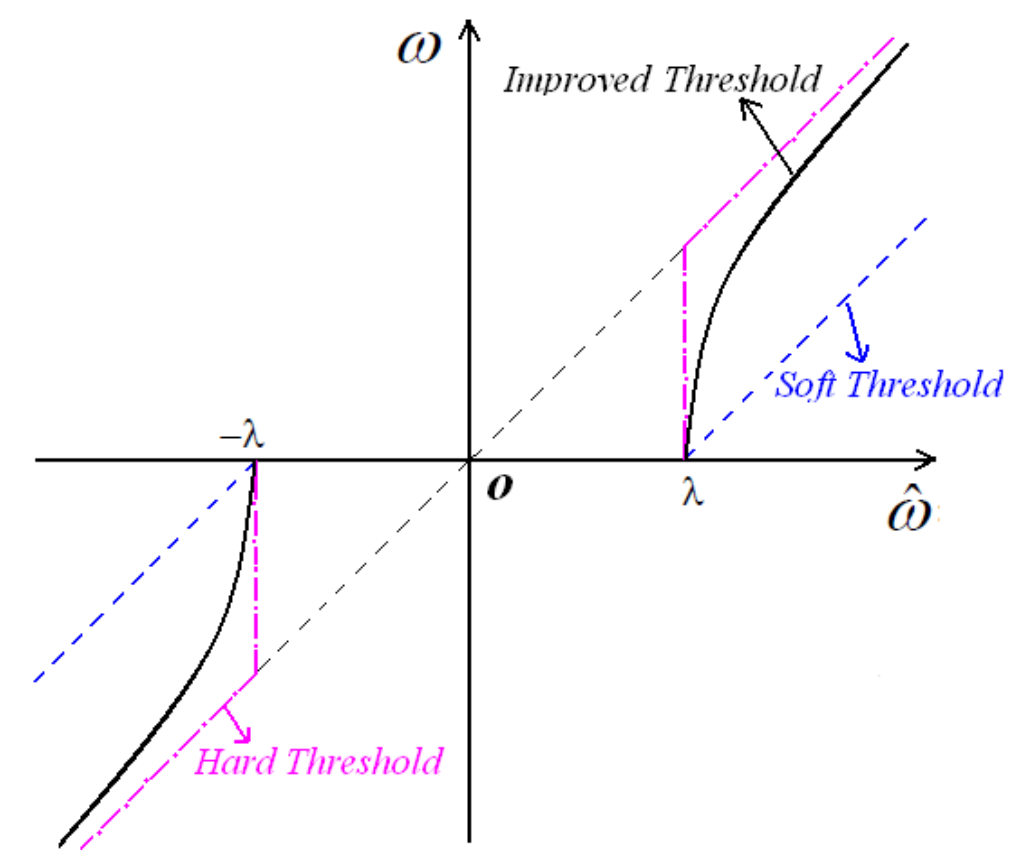

Fig. 1 Three threshold functions' schematic diagram

\section{Experimental Results}

In this paper, we take the square-wave signal (Fig. 2 (a)) for example. First of all, we add Gaussian white noises to the square-wave signal (Fig. 2 (b)), using the traditional hard-threshold and soft-threshold methods de-noise the noisy signal, the de-noised signals are show in Fig. 2 (c) and (d) respectively. Then decompose the noisy signal by EMD method, obtain the IMF indifferent scales (Fig. 3).Here, we de-noise the first three IMF components by using the hard-threshold and the soft-threshold functions as well as the improvement threshold function respectively, the threshold value is set adaptive by each scale IMF component respectively, the de-noised signal are shown in Fig. 2 (e) $\sim(\mathrm{g})$. 
The signal-to-noise ratio (SNR) and mean square error (MSE) which estimated by the different wavelet threshold methods are shown in Table 1 by Eq. 7 and Eq. 8[4].
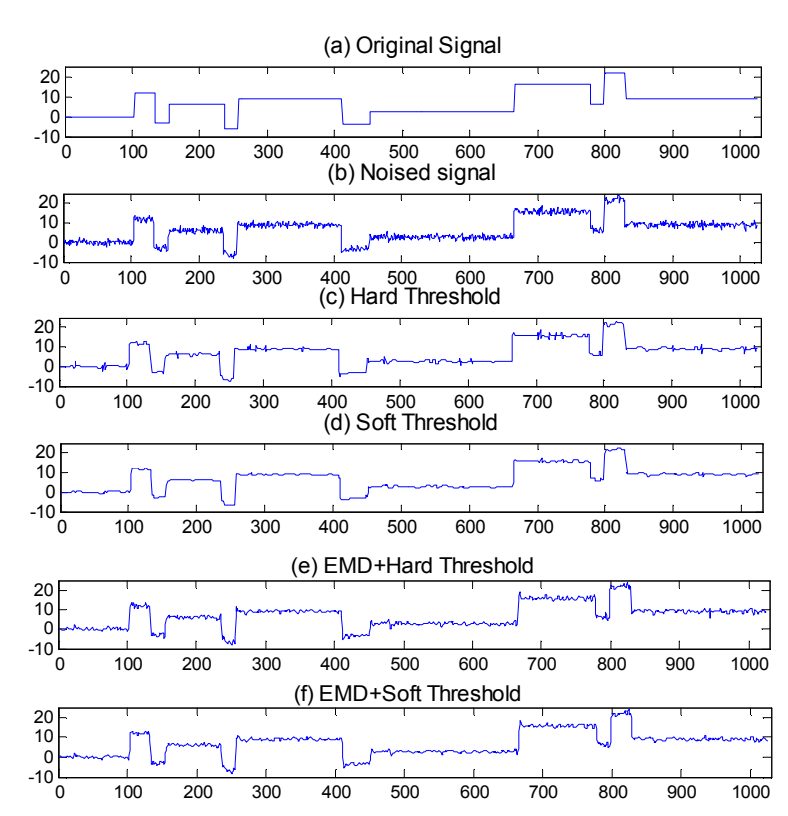

(g) EMD+Improved Threshold

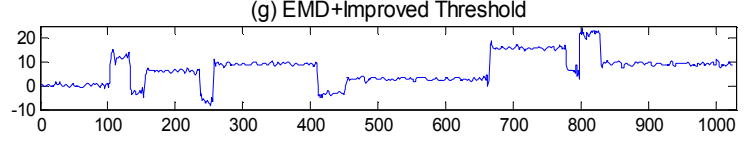

Fig. 2 Denoised signal by different methods

$$
\begin{aligned}
& S N R=10 \lg \left(\sigma_{x}^{2} / \sigma_{e}^{2}\right) . \\
& M S E=\frac{1}{N} \sum_{i=1}^{N}(y(i)-\hat{y}(i))^{2} .
\end{aligned}
$$

Where $\sigma_{x}^{2}$ is the purified signal variance, $\sigma_{e}^{2}$ is the noise variance, y(i) is the de-noised signal, $\hat{y}(i)$ is the actual signal.

Table 1 Comparison of de-noising results under the different methods

\begin{tabular}{|c|c|c|c|c|c|}
\hline & $\begin{array}{c}\text { Hard } \\
\text { Threshold }\end{array}$ & $\begin{array}{c}\text { Soft } \\
\text { Threshold }\end{array}$ & $\begin{array}{c}\text { EMD+Hard } \\
\text { (3 layers) }\end{array}$ & $\begin{array}{c}\text { EMD+Soft } \\
\text { (3 layers) }\end{array}$ & $\begin{array}{c}\text { EMD +Improved } \\
\text { Function (3 layers) }\end{array}$ \\
\hline SNR [dB] & 21.3213 & 20.1386 & 21.2932 & 20.6602 & 21.5154 \\
\hline MSE & 0.3608 & 0.2211 & 0.2108 & 0.2003 & 0.1924 \\
\hline
\end{tabular}

From the Table1, it can be clearly seen that the de-noised signal by the EMD and improved threshold function is better than others. 


\section{Summary}

The EMD can de-noise the nonlinear and non-stationary noisy signal effectively. Also, the weak occasional and periodic impulse signals can be extracted directly by using EMD method.

The new threshold function inferred in the paper can obtain more detail original signal information, and avoid the oscillation effectively, so that the de-noised signal can be much more closely to the original signal.

Combined with the new threshold function and EMD method, the de-noised signal can be more realistic approximation of the original signal.

\section{Acknowledgement}

This research is supported by the projects (60804025) of the National Natural Science Foundation of China. It is also supported by the project (2008555) of education department of Liaoning province. All the support is appreciated.

\section{References}

[1] C. Sidney Burrus, Ramesh A. Gopinath, H.T. Guo, Introduction to Wavelets and Wavelet Transforms. China Machine Press, 2008.

[2] Russell J C, Lardner T J, Experimental determination of frequencies and tension for elastic cables [J]. Journal of Engineering Mechanics. (1998) 1067-1072.

[3] Z.X. Chen, J.W. Xu, D.B. Yang, L.J. Zhang, Weak Signal Detection and Extraction Against the Background of Chaotic Noise Based on Empirical Mode Decomposition. Mechanical Science and Technology. (2006) 220-224.

[4] M. Han, Y.H. Liu, J.H. Xi, Z.W. Shi: Chaotic Signal Denoising Based on Threshold Selection of Wavelet Transform. Information and Control. (2005) 543-547.

[5] Y.K.Sun, Wavelet Analysis and Its Applications. China Machine Press, 2005. 